\title{
Mise en œuvre des composantes du modèle ssp pour la PE / E au Nigéria : une analyse des coûts
}

\author{
Pooja Sripad \\ Population Council \\ Sara Chace Dwyer \\ Population Council \\ Gloria Adoyi \\ Population Council
}

Follow this and additional works at: https://knowledgecommons.popcouncil.org/departments_sbsr-rh

Part of the Demography, Population, and Ecology Commons, Family, Life Course, and Society Commons, International Public Health Commons, Maternal and Child Health Commons, and the Medicine and Health Commons

How does access to this work benefit you? Let us know!

\section{Recommended Citation}

Sripad, Pooja, Sara Chace Dwyer, and Gloria Adoyi. 2019. "Mise en œuvre des composantes du modèle ssp pour la PE / E au Nigéria : une analyse des coûts," Ending Eclampsia Country Brief. Washington, DC: Population Council. 


\section{MISE EN CEUVRE DES COMPOSANTES DU MODĖLE SSP POUR LA PE / E AU NIGÉRIA : UNE ANALYSE DES COÛTS}

\section{INTRODUCTION}

Le taux de mortalité maternelle au Nigéria est de 814 pour 100.000 naissances vivantes [1]. La pré-éclampsie et l'éclampsie (PE / E) constituent la quatrième cause de mortalité maternelle au Nigéria, comptant pour $11 \%$ des décès maternels [2]. La PE est identifiée par une hypertension artérielle et la présence de protéines dans l'urine des femmes enceintes après 20 semaines de gestation. Les femmes atteintes de PE courent un risque accru de dommages / défaillances d'organes, d'accouchements prématurés, de perte de grossesse et d'accident vasculaire cérébral. La PE peut évoluer jusqu'à l'éclampsie, caractérisée par des convulsions, et peut être associée à des dommages des reins et du foie, ainsi qu'au décès maternel. Les risques pour le bébé comprennent la naissance prématurée, un faible poids à la naissance et la mortinatalité et la mort, entre autres [3]. Les bébés nés avant terme à cause d'une PE sont plus à risque de problèmes de santé à long terme. Les risques de PE / E peuvent être atténués par un dépistage régulier au cours des soins prénatals et pendant la période postnatale. Une fois détectée, une surveillance régulière de la $P E$ peut réduire la progression vers une $P E$ / $E$ sévère. La $P E$ / $E$ sévère elle-même peut être prise en charge par l'administration de sulfate de magnésium (MgSO4) et d'antihypertenseurs [3].

Entre 2016 et 2018, Population Council, en partenariat avec les Ministères de la Santé Fédéraux et des États du Nigéria, a mis en œuvre une intervention visant à lutter contre la PE / E dans trois États: Ebonyi, Cross River et Kogi. C'était dans le cadre du projet Ending Eclampsia, un investissement quinquennal de l'USAID qui a mis en œuvre des aspects du modèle de soins de santé primaires (SSP) pour la PE / E au Bangladesh, au Nigeria et au Pakistan.

L'intervention au Nigéria comprenait cinq composantes du modèle de SSP pour la PE / E: (1) le partage des tâches avec des prestataires de SSP en vue de détecter et de prendre en charge la PE / E (MgSO4 et référence) ; (2) l'introduction de la fourniture de médicaments antihypertenseurs au niveau des SSP; (3) la mobilisation des groupes de femmes en vue d'accroître l'utilisation des soins prénatals; (4) la formation des agents logistiques en vue de réduire les ruptures de stock de produits de PE / E; et (5) les messages d'éducation sanitaires.

Ce bref rapport décrit le coût réel de la mise en œuvre de chaque composante au Nigeria. Des descriptions complètes de l'intervention sont disponibles sur le site Internet de_Ending Eclampsia.

\section{Modèle de Soins de Santé Primaires pour la PE/E}

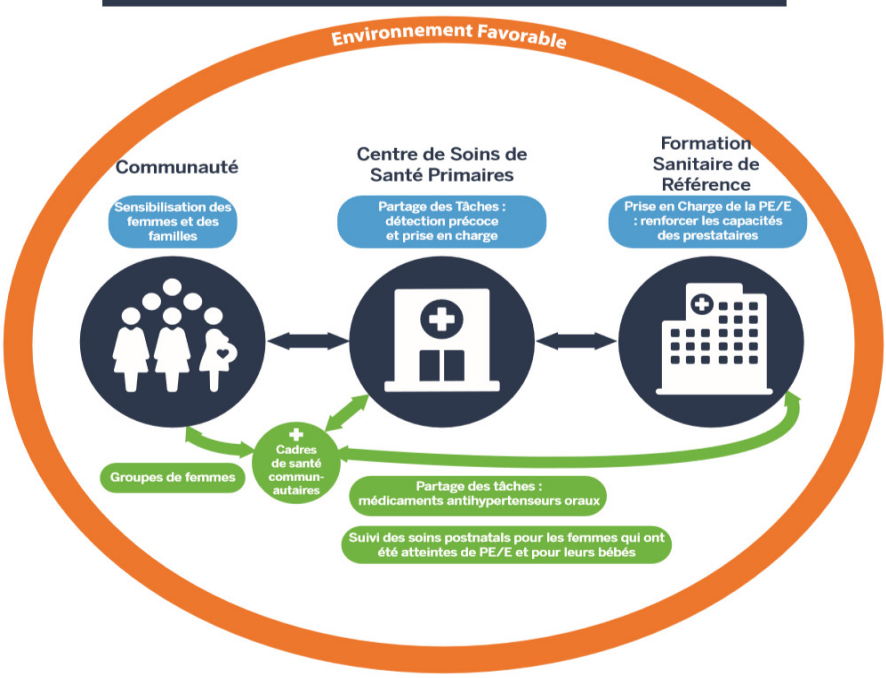

\section{GRANDES LIGNES}

- Composante 1 - Partage des tâches principales pour la détection et la prise en charge de la PE / E au niveau des SSP: 588 prestataires de 180 formations sanitaires réparties dans 3 États ont été formés, pour un total de 271.909 USD et 462 USD par prestataire formé. Le coût réel par prestataire a varié.

- Composante 2 Les médicaments antihypertenseurs au niveau des SSP: 80 prestataires formés dans 40 formations sanitaires réparties dans 2 états, pour un total de 34.048 USD et 426 USD par prestataire formé.

- Composante 3 Les groupes de femmes pour accroître l'utilisation des soins prénatals : 48 femmes leaders formées pour un total de 18.035 \$ et 376 \$ par dirigeante formée.

- Composante 4 Formation des agents logistiques : 72 agents logistiques formés pour un total de 15.869 \$ et 220 \$ par agent formé.

- Composante 5 Messages d'éducation sanitaire : 180 prestataires formés pour un total de 96.446 USD et 536 USD par prestataire.

- Les estimations incluent les coûts payés par le projet, les dons des ministères de la santé au niveau fédéral et des États, ainsi que les coûts d'opportunité pour les participants. Environ $18 \%$ des coûts totaux ont été couverts par le gouvernement et le peuple du Nigéria.

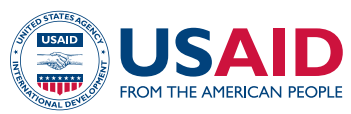

Rendu possible grâce au généreux soutien du peuple américain par l'intermédiaire de l'Agence des ÉtatsUnis pour le Développement International (USAID)
Population Council mène des recherches et propose des solutions qui améliorent la vie dans le monde entier. De grandes idées appuyées par des données probantes : C'est notre modèle pour un changement mondial. popcouncil.org

(c) 2019 The Population Council, Inc
POPULATION COUNCIL

Ideas. Evidence. Impact. 


\section{ANALYSE DES COÛTS}

Au Nigéria, le projet Ending Eclampsia a collecté des données rétrospectives sur les coûts entre mars et mai 2019. Une approche par ingrédients de coût a été utilisée. Chaque élément (entrée) requis pour la mise en œuvre des activités du modèle de SSP pour la PE / E a d'abord été identifié, puis regroupé dans des catégories de coûts : a) le personnel, b) les fournitures, c) les infrastructures ; d) les déplacements ; et (e) les divers. Le coût économique de chaque activité a été calculé indépendamment du fait que le projet ait directement payé pour les biens ou les services. Par exemple, pour prendre en compte le coût d'opportunité des prestataires pour la participation à une formation, leur salaire journalier a été calculé même s'ils n'étaient pas compensés par le projet. Les coûts de gestion du projet ont également été saisis.

Les données sur les coûts ont été collectées à partir des budgets et des rapports de dépenses du projet Ending Eclampsia. Lorsque les informations sur les coûts n'étaient pas disponibles (par exemple, les tarifs journaliers), des estimations moyennes ont été fournies par les représentants des Ministères et par les agents de santé des différents États exécutant les interventions. Sur la base des enseignements tirés de la mise en œuvre du projet, des coûts supplémentaires ont été ajoutés dans certains cas. Par exemple, le nombre de visites de supervision a été standardisé dans les trois États.

Les données sur les coûts ont été généralement collectées en Naira nigérian au cours de l'année de l'achat. En raison des variations de prix par État, des données sur les coûts ont été collectées dans chaque État. Une moyenne de trois États a été utilisée pour estimer le coût total avant d'être convertie en dollars des États-Unis d'Amérique (USD) de 2018 en utilisant le taux de change de clôture du 31 décembre qui était de 363,61 Nairas nigérians (NGN) pour 1 USD (tableau de changes XE). Les coûts de gestion du programme et de bureau ont été collectés en USD et convertis en Nairas nigérians en cas de nécessité, en utilisant le même taux de change. Les coûts par participant ont été calculés pour chaque composante.

\section{COMPOSANTE : 1 - PARTAGE DES TÂCHE PRINCIPALES AU NIVEAU DES SSP}

La composante 1 reflète le partage des tâches de prévention, de détection et de prise en charge de la PE / E au niveau des soins de santé primaires. Il y avait quatre activités principales : (1) la formation des prestataires de soins de santé secondaires sur la détection et la prise en charge de la PE / E; (2) la formation des prestataires de SSP sur l'administration d'une dose de charge de MgSO4 et sur les références; (3) la formation des agents médicaux en charge des SSP sur la détection et la prise en charge de la PE / E; et (4) le soutien à la supervision régulière des prestataires au niveau des formations sanitaires.

Le tableau 1 présente les coûts de reproduction du partage de tâches de la Composante 1 au niveau des soins de santé primaires. Le coût total a été de 271.909 dollars (98.868.694 NGN), les catégories de coûts les plus importantes étant le personnel, suivies des déplacements et des infrastructures. Sur les 138.000 USD de coûts de TABLEAU 1: Coût de la composante de partage des tâches principales au niveau des SSP

\begin{tabular}{|l|c|c|c|c|c|}
\hline \multicolumn{1}{|c|}{ Éléments } & $\begin{array}{c}\text { Cross River } \\
(\text { USD) }\end{array}$ & $\begin{array}{c}\text { Ebonyi } \\
(\text { USD })\end{array}$ & $\begin{array}{c}\text { Kogi } \\
(\text { USD })\end{array}$ & Coût Total (USD) & Coût Total (NGN) \\
\hline Personnel* & $\$ 52,481$ & $\$ 37,635$ & $\$ 44,922$ & $($ USD) & Coût Total (NGN) \\
\hline Fournitures & $\$ 910$ & $\$ 686$ & $\$ 783$ & $\$ 2,379$ & 864,988 \\
\hline Infrastructures* & $\$ 16,303$ & $\$ 15,346$ & $\$ 16,693$ & $\$ 49,377$ & $1,795,3893$ \\
\hline Déplacements & $\$ 38,180$ & $\$ 23,687$ & $\$ 18,231$ & $\$ 80,099$ & $2,9124,640$ \\
\hline Divers & $\$ 693$ & $\$ 600$ & $\$ 641$ & $\$ 1,934$ & 703,320 \\
\hline Total & $\$ 108,568$ & $\$ 77,955$ & $\$ 81,270$ & $\$ 271,909$ & $98,868,694$ \\
\hline
\end{tabular}

* Le total inclut les estimations de la gestion globale du programme fournies par Abuja

FIGURE 1: Coût par participant pour chaque activité de la Composante 1: Partage des tâches principales au niveau des soins de santé primaires

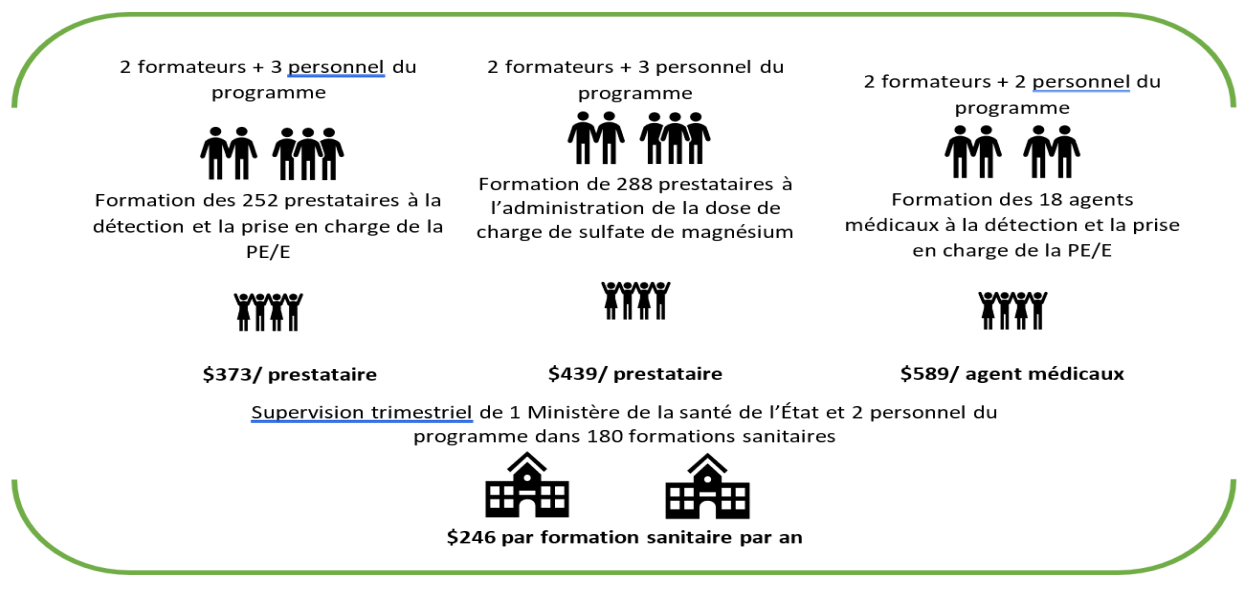


personnel, environ 51.407 USD (37\%) ont été donnés en nature par des ministères de la Santé des États. La figure 1 présente la répartition des coûts par participant. Au total, 558 prestataires de 180 formations sanitaires ont été formés dans les trois États. Pour chaque activité, les participants ont été formés pendant deux jours, puis ont participé à une formation de recyclage de deux jours. Les activités étaient dirigées par des formateurs certifiés, le personnel du programme et divers employés des ministères fédéraux et des États. Le coût moyen par prestataire était de 462 dollars (168.114 NGN). Toutefois, le coût par prestataire variait par activité. Dans l'activité 1, 252 prestataires issus de formations sanitaires secondaires ont été formés à la détection et à la prise en charge de la PE / E pour une moyenne de 373 USD par prestataire. Pour l'activité 2, 288 prestataires issus de formations sanitaires primaires ont été formés à la détection de la PE / E et à l'administration d'une dose de charge de MgSO4 pour une moyenne de 439 USD par prestataire. Dix-huit agents médicaux des CSPs de l'État de Cross River ont été formés à la détection précoce et à la prise en charge de la PE / E pour une moyenne de 589 \$ par prestataire. Enfin, une équipe du personnel des Ministères de la Santé des États et de Population Council a effectué des visites de supervision trimestrielles dans 180 formations sanitaires. Le coût par formation sanitaire était de 246 \$.

En moyenne, le coût total dans l'État d'Ebonyi était similaire à celui de l'État de Kogi. Globalement, le coût total dans l'État de Kogi était supérieur de 3.314,71 USD à celui de l'État d'Ebonyi. Les coûts dans l'État de Cross River étaient plus élevés que ceux d'Ebonyi et de Kogi car ils compreTABLEAU 2 : Coût de l'introduction de la composante relative aux antihypertenseurs aux SSP

\begin{tabular}{|l|c|c|c|c|}
\hline \multicolumn{1}{|c|}{ Éléments } & Ebonyi (USD) & $\begin{array}{c}\text { Kogi } \\
(\text { USD) }\end{array}$ & Coût Total (USD) & $\begin{array}{c}\text { Coût Total } \\
(\mathrm{NGN})\end{array}$ \\
\hline Personnel* & $\$ 7,243$ & $\$ 7,633$ & $\$ 17,246$ & 6270790 \\
\hline Fournitures & $\$ 124$ & $\$ 124$ & $\$ 248$ & 90000 \\
\hline Infrastructures* & $\$ 1,576$ & $\$ 1,993$ & $\$ 4,604$ & 1674093 \\
\hline Déplacements & $\$ 6,108$ & $\$ 5,266$ & $\$ 11,374$ & 4135760 \\
\hline Divers & $\$ 281$ & $\$ 295$ & $\$ 576$ & 209480 \\
\hline Total & $\$ 15,332$ & $\$ 15,311$ & $\$ 34,048$ & $12,380,122$ \\
\hline
\end{tabular}

* Le total inclut les estimations de la gestion globale du programme fournies par Abuja

FIGURE 2 : Coût par participant au titre de la Composante 2 introduisant les antihypertenseur aux SSP

2 formateurs +3 personnel du programme

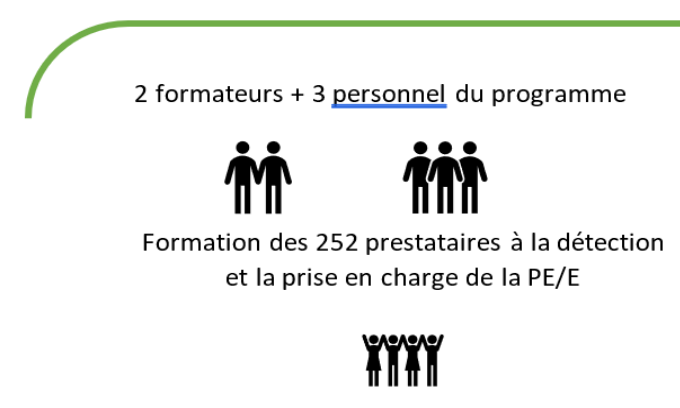

Formation des 252 prestataires à la détection et la prise en charge de la PE/E

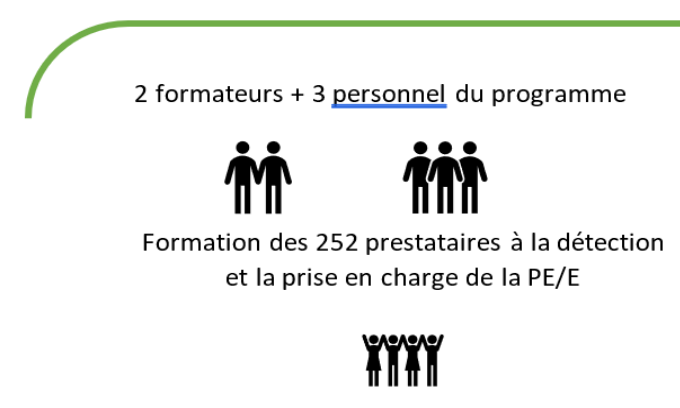

$\$ 241 /$ prestataire naient la formation des Agents Médicaux ainsi que les coûts administratifs comparativement plus élevés de l'État.

\section{COMPOSANTE 2 : INTRODUCTION DE MÉDICAMENTS ANTIHYPERTENSEURS AUX SSP}

Ending Eclampsia a introduit le partage des tâches relatif à la fourniture du médicament antihypertenseur (méthyldopa oral) au niveau des SSP dans les États d'Ebonyi et de Kogi. Cela comprenait la formation des prestataires au counseling des clients sur les signes de la PE / E et à l'administration des antihypertenseurs. Le tableau 2 indique le coût total de la mise en œuvre de la composante relative aux antihypertenseurs dans deux États. Le coût total de la mise en œuvre de la composante relative aux antihypertenseurs était de 34.048 USD (12.380.122 NGN), soit un coût moyen par fournisseur de 426 USD (154.752 NGN). Sur les 17.245 dollars de dépenses liées au personnel, 4.480 dollars (26\%) ont été donnés en nature par les Ministères de la Santé des États. La figure 2 indique la répartition des coûts par prestataire. Au total, 80 prestataires de 40 formations sanitaires ont participé à une formation de deux jours. Les formations ont été menées par deux formateurs et trois membres du personnel du programme, avec le soutien d'environ six membres du personnel des MSE. Le coût par prestataire était de 241 dollars (87.630 NGN). Quarante des formations sanitaires ayant participé à la composante relative aux antihypertenseurs ont également bénéficié d'un appui à la supervision pour l'administration d'antihypertenseurs. Cela s'est produit lors des mêmes visites de supervision décrites dans la composante
1 Ministère de la santé de l'État +2 personnel du programme

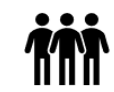

Supervision trimestrielle de 40 formations

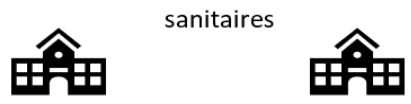

$\$ 189$ par formation sanitaire par an

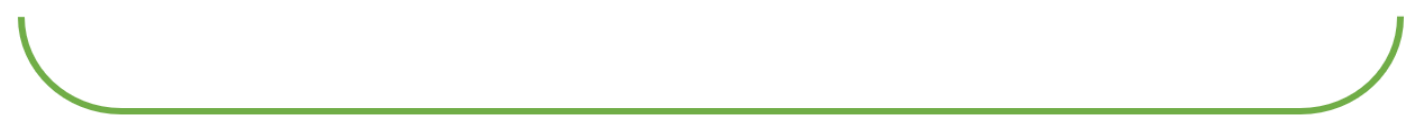


1 et les coûts ont été partagés entre les deux composantes. Le coût total par formation sanitaire pour la supervision au titre de cette composante a été de 189 USD (68.722 NGN).

\section{COMPOSANTE 3: LES GROUPES DE FEMMES POUR ACCROÎTRE L'UTILISA- TION DES SOINS PRÉNATALS}

Pour encourager l'utilisation des services de soins prénatals et de soins postnatals dans les formations de santé de l'État de Cross River, des responsables de groupes de femmes ont été formées à la diffusion à leurs pairs d'informations de santé concernant les soins prénatals et postnatals. Elles ont été spécifiquement formées à l'utilisation de supports de formation illustrées afin de partager des messages de santé avec les femmes de leur communauté et d'un régistre pour enregistrer les activités. Après la formation, elles ont partagé des messages de santé lors de réunions mensuelles de groupes de femmes. Les femmes leaders ont également bénéficié d'un soutien au mentorat de la part d'un(e) infirmier(ère) en chef désigné(e) par le MSE. Le tableau 3 souligne le coût total de la mise en œuvre du modèle relatif aux groupes de femmes.

TABLEAU 3 : Coût de la composante relative aux groupes de femmes dans l'État de Cross River

\begin{tabular}{|l|c|c|}
\hline \multicolumn{1}{|c|}{ Éléments } & Naira (NGN) & Dollar (USD) \\
\hline Personnel* & $2,117,500$ & $\$ 5,824$ \\
\hline Fournitures & 54,000 & $\$ 149$ \\
\hline Infrastructures* & $1,612,500$ & $\$ 4,435$ \\
\hline Déplacements & $2,706,000$ & $\$ 7,442$ \\
\hline Divers & 67,800 & $\$ 186$ \\
\hline Total & $6,557,800$ & $\$ 18,035$ \\
\hline
\end{tabular}

* Le total inclut les estimations de la gestion globale du programme fournies par Abuja

Le total a été de 18.035 dollars (6.557.800 NGN). Au total, 48 femmes leaders ont été formées pour un coût de 376 dollars par leader. Les formations ont été menées par un formateur, deux membres du personnel du programme et deux membres du personnel du MSE. Les visites de supervision ont été effectuées par le personnel du MSE et du programme.
En moyenne, chaque femme leader a touché 250 à 300 femmes. Les ministères de la santé des États et les femmes leaders ont donné en nature environ 3.905 dollars de leur temps et de leur utilisation des espaces communautaires. La figure 3 indique la répartition des coûts par participant pour groupe de femmes.

FIGURE 3 : Coût par participante au titre de la Composante 3 - groupes de femmes pour accroître l'utilisation des services de soins prénatals

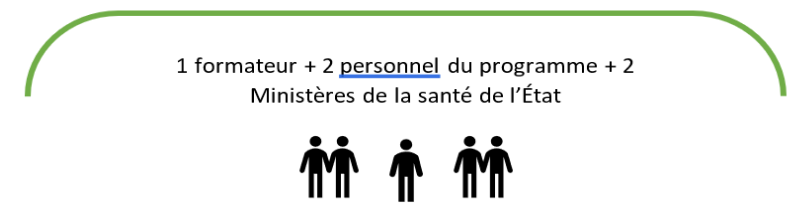

Formation et mentorat pour 48 prestataires et leaders de groupes de femmes

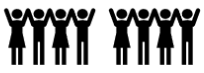

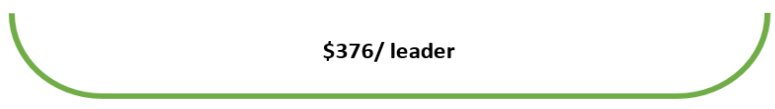

\section{COMPOSANTE 4 : AGENT LOGISTIQUE FORMATION}

Lors de la mise en œuvre de la composante 1, l'équipe de Ending Eclampsia a identifié des lacunes au niveau de la communication entre les formations sanitaires et les pharmacies. Cela a laissé des formations sanitaires sans le MgSO4 et la méthyldopa orale nécessaires à la prise en charge des cas de PE / E. Soixante-douze agents logistiques de 36 formations sanitaires secondaires ont reçu une formation supplémentaire sur la gestion de la chaîne d'approvisionnement. Le tableau 4 détaille le coût total de la Composante 4.

Le coût total de la mise en œuvre de la Composante 4 a été de 15.869 dollars (5.036.598 NGN) et de 220 dollars par agent logistique. Sur les 6.179 dollars de dépenses liées au personnel, 3.905 dollars (27\%) ont été donnés en nature par les ministères de la santé des États.

TABLEAU 4 : Coût de la composante relative à la formation des agents logistiques

\begin{tabular}{|l|c|c|c|c|c|}
\hline \multicolumn{1}{|c|}{ Éléments } & Cross River (USD) & $\begin{array}{c}\text { Ebonyi } \\
(U S D)\end{array}$ & $\begin{array}{c}\text { Kogi } \\
(\text { USD) }\end{array}$ & Coût Total (USD) & Coût Total (NGN) \\
\hline Personnel* & $\$ 1,185$ & $\$ 1,253$ & $\$ 1,372$ & $\$ 6,180$ & $1,887,698$ \\
\hline Fournitures & $\$ 0$ & $\$ 0$ & $\$ 0$ & $\$ 0$ & 0 \\
\hline Infrastructures* & $\$ 721$ & $\$ 721$ & $\$ 748$ & $\$ 3,224$ & 796,000 \\
\hline Déplacements & $\$ 2,178$ & $\$ 2,178$ & $\$ 2,013$ & $\$ 6,369$ & $2,316,000$ \\
\hline Divers & $\$ 34$ & $\$ 34$ & $\$ 28$ & $\$ 96$ & 36,900 \\
\hline Total & $\$ 4,117$ & $\$ 4,185$ & $\$ 4,162$ & $\$ 15,869$ & $5,036,598$ \\
\hline
\end{tabular}

* Le total inclut les estimations de la gestion globale du programme fournies par Abuja 
La figure 4 indique la répartition des coûts par participant pour la formation des agents logistiques.

FIGURE 4 : Coût par participant pour la Composante 4 relative à la formation des agents logistiques

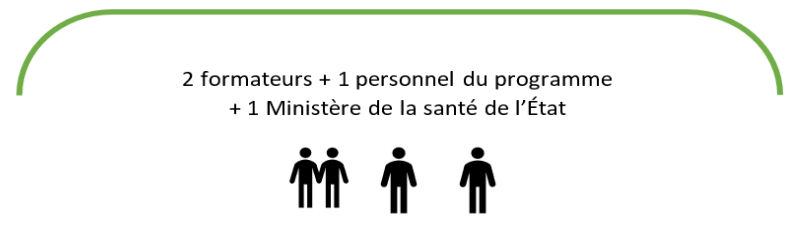

Formation pour 72 agents logistique

\section{martinger}

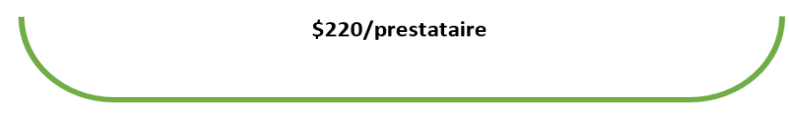

\section{COMPOSANTE 5 : MESSAGES DE SANTÉ AVANT LES SOINS PRENATAUX}

La composante 5 visait à préparer les prestataires des formations sanitaires primaires et secondaires à fournir aux femmes des messages éducatifs sur la PE / E et sur la santé, avant et pendant les visites prénatales. En moyenne, un formateur, trois membres du personnel du programme et un représentant du MSE ont conduit trois formations, une initiale et deux de recyclage, au bénéfice de 180 prestataires dans les trois États.Le coût total de la mise en œuvre de la Composante 5 s'est élevé à 96.446 USD (33.809.907 NGN), comme indiqué dans le Tableau 5, avec un coût moyen par participant de 534 USD (187.833 NGN). Sur les 36.805 dollars de frais de personnel, les MSE ont fait un don en nature d'environ 15.858 dollars (43\%). Le coût de mise en œuvre de cette composante dans l'État de Kogi était moins élevé que dans les deux autres États. Ceci est principalement dû à la réduction des frais de personnel et de déplacement. La figure $\mathbf{5}$ indique la répartition des coûts par participant de la Composante 5 .

\section{CONCLUSION}

Le coût total direct de la mise en œuvre des activités du projet Ending Eclampsia au Nigéria s'est élevé à 436.307

TABLEAU 5 : Coût de la composante relative aux messages de santé

\begin{tabular}{|l|c|c|c|c|c|}
\hline \multicolumn{1}{|c|}{ Éléments } & Cross River (USD) & $\begin{array}{c}\text { Ebonyi } \\
(\text { USD) }\end{array}$ & $\begin{array}{c}\text { Kogi } \\
(\text { USD) }\end{array}$ & Coût Total (USD) & Coût Total (NGN) \\
\hline Personnel* & $\$ 10,437$ & $\$ 13,554$ & $\$ 10,444$ & $\$ 36,806$ & $12,521,107$ \\
\hline Fournitures & $\$ 201$ & $\$ 201$ & $\$ 189$ & $\$ 591$ & 214,800 \\
\hline Infrastructures* & $\$ 5,394$ & $\$ 7,239$ & $\$ 5,459$ & $\$ 19,126$ & $6,578,200$ \\
\hline Déplacements & $\$ 18,771$ & $\$ 11,002$ & $\$ 9,638$ & $\$ 39,412$ & $14,339,500$ \\
\hline Divers & $\$ 171$ & $\$ 171$ & $\$ 171$ & $\$ 512$ & 156,300 \\
\hline Total & $\$ 34,974$ & $\$ 32,167$ & $\$ 25,901$ & $\$ 96,447$ & $33,809,907$ \\
\hline
\end{tabular}

* Le total inclut les estimations de la gestion globale du programme fournies par Abuja
FIGURE 5: Coût par participant au titre de la Composante 5 relative aux messages de santé avant les soins prénatals

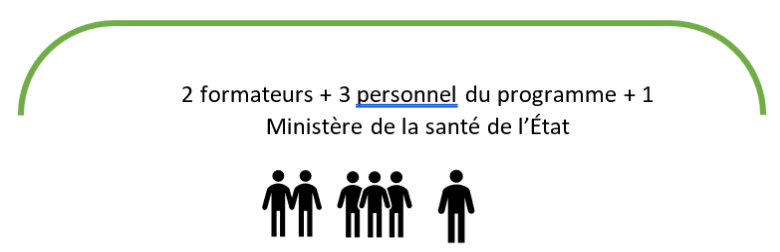

Formation pour 180 prestataires

$\$ 536 /$ prestataire

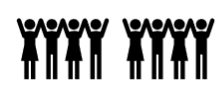

dollars (15.653.121 NGN). Dans toutes les composantes, le personnel et les déplacements ont constitué les catégories de coûts les plus importantes, comptant pour respectivement $47 \%$ et $33 \%$ environ du coût total. Le personnel a constitué la composante de coût la plus importante parce qu'il s'agissait d'une intervention basée sur les ressources humaines. Le projet a directement payé environ $80 \%$ des coûts de personnel enregistrés ici. II était toutefois important d'enregistrer les coûts réels de la contribution des participants au projet. En participant au projet, les prestataires ont été retirés de leurs formations sanitaires, le personnel des MSE n'a pas pu participer à d'autres interventions sanitaires et les femmes leaders ont été détournées d'autres activités vitales et/ ou génératrices de revenus. Par conséquent, cette analyse a permis de capturer les coûts d'opportunité pour les prestataires, le personnel des MSE et les femmes leaders.

Les frais de déplacement étaient élevés parce que le projet avait été mis en œuvre dans trois États et géré par le projet Ending Eclampsia basé à Abuja. Cela a obligé le personnel du programme et les formateurs à voyager fréquemment, entraînant des coûts de transport aérien élevés. Bien qu'il y ait eu des frais de déplacement supplémentaires, le personnel d'Abuja a veillé à une mise en œuvre de qualité des activités dans chaque État. 
Les coûts pouvaient être réduits en embauchant du personnel du programme dans les États, mais des coûts supplémentaires pour l'assurance de la qualité seraient nécessaires.

Étant donné que la mise en œuvre du modèle SSP-EP/E a été mise en œuvre graduellement étape par étape et qu'elle a progressivement ajouté des composantes selon une approche de recherche sur la mise en œuvre qui a testé la faisabilité de la capacité des fournisseurs de SSP à gérer l'EP/E à divers degrés, les coûts pourraient être réduits pour les futurs utilisateurs. Par exemple, si les formations aux messages sur les antihypertenseurs et les soins prénatals étaient initialement intégrées à la composante relative au partage des tâches principales, les coûts de mise en œuvre auraient été partagés et probablement réduits. De même, la mise en œuvre de la formation des agents logistiques et des femmes leaders parallèlement aux ateliers de la Composante Un aurait permis de réduire les frais de déplacement.

Pour reproduire cette intervention, les coûts des infrastructures et des déplacements peuvent varier selon que l'organisation ou l'agence de mise en œuvre a déjà acheté du matériel important et pourrait partager les coûts dans d'autres activités (par exemple : véhicules et projecteurs), ou aura besoin de louer ces biens et services. Les coûts moyens de location ont été utilisés dans cette analyse afin de fournir une synapsis plus vaste du coût, bien que le coût au projet ait pu être plus bas.

Cette analyse des coûts de mise en œuvre sert de point de départ pour ceux qui envisagent de mettre en œuvre différentes composantes du modèle de SSP pour la PE / E. Des moyens de consolider les coûts de mise en œuvre des cinq composantes de l'intervention Ending Eclampsia au Nigéria doivent être explorées. Les travaux futurs pourraient également explorer l'intégration des cinq Composantes dans les mécanismes de formation existants pour les prestataires de soins de santé primaires et la manière d'aider les groupes communautaires à diffuser des messages de santé.

\section{REFERENCES}

1. World Bank Group. WHO, UNICEF, UNFPA, World Bank Group, and the United Nations Population Division. Trends in Maternal Mortality: 1990 to 2015. Geneva, World Health Organization, 2015. World Bank Open Data. https://data.worldbank.org/

2. Frederick Wekesah, Chiamaraoke Izubara. 2017 "Maternal Health in Nigeria: Facts and Figures." Kenya: Africa Population and Health Research Center.

3. World Health Organization. Integrated Management of Pregnancy and Childbirth (IMPAC). https://www.who.int/ maternal_child_adolescent/topics/maternal/impac/en/

\section{CONTACT}

Pooja Sripad, Associate, Population Council

psripad@popcouncil.org

Sara Chace Dwyer, Staff Associate, Population Council schace@popcouncil.org

Gloria Adoyi, Program Officer, Population Council gadoyi@popcouncil.org 